Draft Version November 7, 2018

Preprint typeset using $\mathrm{L}^{A} \mathrm{~T}_{\mathrm{E}} \mathrm{X}$ style emulateapj v. 08/22/09

\title{
COHERENCE OF BURST OSCILLATIONS AND ACCRETION-POWERED PULSATIONS IN THE ACCRETING MILLISECOND PULSAR XTE J1814-338
}

\author{
Anna L. Watts ${ }^{1}$, Alessandro Patruno \& Michiel van der Klis \\ Astronomical Institute "Anton Pannekoek", University of Amsterdam, Kruislaan 403, 1098 SJ Amsterdam, the Netherlands \\ Draft version November 7, 2018
}

\begin{abstract}
X-ray timing of the accretion-powered pulsations during the 2003 outburst of the accreting millisecond pulsar XTE J1814-338 has revealed variation in the pulse time of arrival residuals. These can be interpreted in several ways, including spin-down and wandering of the fuel impact point around the magnetic pole. In this Letter we show that the burst oscillations of this source are coherent with the persistent pulsations, to the level where they track all of the observed fluctuations. Only one burst, which occurs at the lowest accretion rates, shows a significant phase offset. We discuss what might lead to such rigid phase-locking between the modulations in the accretion and thermonuclear burst emission, and consider the implications for spin variation and the burst oscillation mechanism. Wandering of the fuel impact hot spot around a fixed magnetic pole seems the most likely cause for the accretion-powered pulse phase variations. This means that the burst asymmetry is coupled to the hot spot, not the magnetic pole. If premature ignition at this point (due to higher local temperatures) triggers a burning front that stalls before spreading over the entire surface, the resulting localized nuclear hot spot may explain the unusual burst and burst oscillation properties of this source.
\end{abstract}

Subject headings: binaries: general, stars: individual (XTE J1814-338), stars: neutron, stars: rotation, X-rays: bursts, X-rays: stars

\section{INTRODUCTION}

The accreting millisecond X-ray pulsars (AMXPs) are a small class of neutron stars in Low Mass X-ray Binaries that show pulsations in outburst, thought to be caused by magnetic channeling of accreting plasma. Detailed timing studies of these stars reveal diverse behavior that can be interpreted in terms of spin variation or shifts in emission pattern. Both processes may play a role, and the degree to which we can be confident in inferred values of spin-up or spin-down remains a hot topic (Gallowav et al. 2002; Burderi et al. 2006, 2007; Papitto et al. 2007, 2008; Hartman et al. 2008; Riggio et al. 2008).

Some additional way of verifying the timing analysis obtained from the accretion-powered pulsations would be useful, and in this respect two of the AMXPs are particularly valuable. SAX J1808.4-3658 (J1808) and XTE J1814-338 (J1814) also show thermonuclear-powered pulsations, or burst oscillations. These are high frequency variations seen during Type I X-ray bursts, powered by unstable burning of accreted fuel. In these systems the burst oscillation frequency is at (J1814), or very close to (J1808), the spin frequency (Chakrabarty et al. 2003; Strohmayer et al. 2003, hereafter S03). The frequency is stable in the decaying tails of the bursts, with no sign of the large frequency drifts seen in other burst oscillation sources (Muno et al. 2002). In J1814 the frequency is also stable during the rising phase of the bursts, making it the most straightforward candidate for burst oscillation timing (Watts et al. 2005, hereafter W05).

J1814's accretion-powered pulsations show significant pulse time of arrival (TOA) residuals even after correction for orbital Doppler shifts (Papitto et al. 2007, here-

\footnotetext{
${ }^{1}$ A.L.Watts@uva.nl
}

after P07). The cause is still a matter of debate: P07 interpreted the observations in terms of a steady spindown coupled with some jitter due to wandering of the fuel impact hotspot around the magnetic pole. However there are other possibilities, such as changes in beaming due to the accretion shock, that may also lead to the observed variation. In this respect analysis of the burst oscillations may be simpler: although the process responsible is not yet understood, the thermal spectrum suggests a purely surface mechanism, with the accretion shock contributing little to the observed asymmetry.

Some initial investigation of this issue was carried out by S03, who found that burst oscillations in the first 12 bursts were phase-locked to within $2.5^{\circ}$ of the persistent pulsations. Their analysis, however, covered only the first 10 days of the $\approx 50$ day outburst; before any of the variation reported by P07 is apparent. The level of coherence between the two types of pulsations is also important in our efforts to understand the burst oscillation mechanism, which remains mysterious (see the reviews by Strohmaver \& Bildsten 2006, Gallowav et al. 2006). The AMXPs are the only sources in which we can quantify the role of the magnetic field. We want to know, for example, whether the burst oscillations couple to the magnetic field or to the fuel stream impact point.

\section{TIMING ANALYSIS}

J1814 was discovered in 2003 in the Rossi X-ray Timing Explorer (RXTE) Galactic bulge monitoring campaign (Markwardt \& Swank 2003), and remained in outburst for nearly 2 months. The pulsar has a spin frequency of $314.4 \mathrm{~Hz}$ and resides in a binary with an orbit of 4.3 hours (Markwardt et al. 2003). During the outburst over $425 \mathrm{ks}$ of high time resolution data were taken with $R X T E$ 's Proportional Counter Array (PCA, Jahoda et al. 2006). A total of $28 \mathrm{X}$-ray bursts were de- 
tected, all with burst oscillations at the spin frequency. Both accretion-powered pulsations and burst oscillations have a strong overtone at twice the spin frequency (S03).

For our timing analysis we use all available pointed observations from the $R X T E$ PCA with Event mode data (time resolution $122 \mu \mathrm{s}, 64$ binned energy channels) or Good Xenon mode data (time resolution $1 \mu \mathrm{s}, 256 \mathrm{un}-$ binned energy channels). The latter were rebinned in time to $122 \mu$ s time resolution. Data were barycentered using the JPL DE405 ephemeris and a spacecraft ephemeris including fine clock corrections which together provide an absolute timing accuracy of 3-4 $\mu$ s (Rots et al. 2004), using the source position of Krauss et al. (2005).

In analysing the accretion-powered pulsations we discard the X-ray bursts, removing all data from $50 \mathrm{~s}$ before to $200 \mathrm{~s}$ after the burst rise, and select only photons in the $2.5-17 \mathrm{keV}$ range to maximize the signal to noise ratio. Pulse profiles are built using the fixed frequency solution of P07 to fold segments of approximately $500 \mathrm{~s}$ of data after subtracting the background contamination (using the FTOOL pcabackest). The TOAs were then obtained by cross-correlating the folded profiles with a pure sinusoid whose frequency represents the spin frequency of the neutron star. The same procedure is repeated for the first overtone. The fiducial point used in measuring the TOAs was the peak of the sine wave being crosscorrelated. The determination of pulse TOAs and their statistical uncertainties follows the standard radio pulsar technique (Taylor 1993). Fitting a Keplerian orbit plus a constant spin frequency $\nu$, or spin derivative $\dot{\nu}$, we obtain solutions consistent with those of P07.

Like P07, we find a strong anti-correlation between flux and the accretion-powered pulse TOA residuals. It is interesting, however, that this anti-correlation becomes weaker when one considers the residuals from an ephemeris that includes a constant $\dot{\nu}$ term. A rank correlation test between flux and residuals for a constant frequency model gives a Spearman coefficient of $\rho=-0.71$ (fundamental) and $\rho=-0.90$ (first overtone), with a probability of $<0.01 \%$ that the two variables are not anti-correlated. Including spin-down, the magnitudes of the Spearman coefficients fall to $\rho=-0.56$ (probability still $<0.01 \%$ that the two variables are not anticorrelated, but larger than the probabilities for the zero spin derivative case) and $\rho=-0.12$ (a probability of $27 \%$ that the two variables are not anti-correlated) respectively. As we will argue in Section 3. an accretion-rate dependent hot spot location may be able to explain the entire residual record, with no need for spin variation.

We then apply the same timing procedure to the X-ray bursts. As in W05 and Watts \& Strohmaver (2006) we use data where the count rate is at least twice the preburst level. For the first 27 bursts there is no evidence of frequency variability during the bursts (W05), so we use the entire burst to generate a folded profile. The final burst requires more care, as there is a statistically significant frequency drop in the late stages of the burst rise (Fig.20 of W05). For this burst we use only the first $2 \mathrm{~s}$ of the burst rise, before the frequency starts to shift. For each folded burst profile we then compute residuals using the same ephemeris that we used for the accretionpowered pulsations. Again we cross-correlate the folded profile using both a fundamental and an overtone. Figure 1 shows the TOA residuals for the accretion-powered

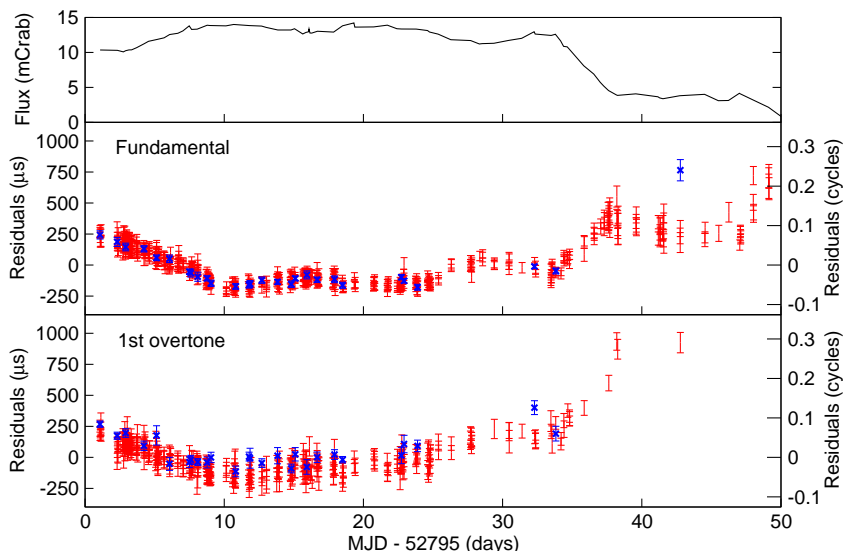

FIG. 1.- Phase residuals for the accretion-powered pulsations (bars; red) and the burst oscillations (crosses; blue), compared to a model that has a constant frequency and orbital Doppler shifts. The final burst, which has no detectable 1st overtone component, requires special treatment; see text. The units on the right axis are rotational cycles. The top panel shows the outburst light curve.

pulsations and the burst oscillations.

To check whether the TOAs of the burst oscillations are consistent with having the same temporal dependence as the accretion-powered pulsations we fit a constant frequency model to the two TOA sets separately (excluding the final burst, see below). The fitted frequency is the same within the statistical uncertainties both for the fundamental and the first overtone. We also tried fitting the two TOA sets with a spin frequency plus a frequency derivative. Again the two solutions are consistent within the statistical uncertainties. However P07 already noted that both ephemerides are a poor fit to the accretionpowered pulse TOA residuals, and the same is true for the burst oscillation residuals. They are formally inconsistent with a constant frequency model: for the fundamental this assumption gives a $\chi^{2}$ of 459 for 23 degrees of freedom (dof). They are also inconsistent with a constant $\dot{\nu}$, as $\chi^{2}$ in this case is still large, 80 for 22 dof. Similar results are obtained for the first overtone.

It is clear from the Figure that, for all but the final burst, the burst oscillation TOA residuals track the accretion pulsation residuals. To test the phase-lock we computed the phase difference $\Delta \phi_{m}=\nu\left[\mathrm{TOA}_{\mathrm{bur}}-\right.$ TOA $\left._{\text {acc }}\right]$, using the accretion pulse TOA residuals from the observation containing each burst. We then fitted a constant. For the fundamental we found a $\chi^{2}$ of 15.3 (26 dof), with best fit $\Delta \varphi_{m}=(0.004 \pm 0.002)$ rotational cycles. For the first overtone we found a $\chi^{2}$ of 11.3 (26 dof), with best fit $\Delta \varphi_{m}=-(0.001 \pm 0.003)$ rotational cycles. The fact that a constant $\Delta \varphi_{m}$ is such a good fit confirms that the two sets of pulsations are phase-locked. The small $(2 \sigma)$ non-zero offset in the fundamental bears comment. Unlike the burst oscillations, the accretion-powered pulsations have soft lags across the $2.5-20 \mathrm{keV}$ band, with higher energy photons arriving earlier in phase (Watts \& Strohmayer 2006). Taking this into account, we find that the burst oscillation TOAs are completely coincident with the softer $(2.5$ $5 \mathrm{keV}$ ) component of the accretion pulsations, thought to originate from stellar surface (Section [3). The only exception to the phase-locking is the final burst, with $\Delta \varphi_{m}=(0.2 \pm 0.04)$ rotational cycles.

When calculating burst oscillation residuals some care 
is required, since if accretion continues during the burst, there might still be a contribution from the accretionpowered pulsations. The resulting bias in the burst oscillation phase can be calculated easily by considering the profile that results from the addition of two offset sinusoidal profiles. Standard trigonometric identities yield a relation between $\Delta \varphi_{m}$ (the measured offset between burst oscillation phase and accretion-powered pulse phase) and $\Delta \varphi_{b}$ (the bias, i.e. the offset between measured and true burst oscillation phase caused by residual accretion):

$$
\tan \Delta \varphi_{m}=\frac{\sin \left(\Delta \varphi_{m}-\Delta \varphi_{b}\right)}{\left[N_{\mathrm{acc}} r_{\mathrm{acc}} / N_{\mathrm{bur}} r_{\mathrm{bur}}\right]+\cos \left(\Delta \varphi_{m}-\Delta \varphi_{b}\right)}
$$

where $N_{\text {acc }}$ and $N_{\text {bur }}$ are the number of accretion and burst photons in the folded profile, $r_{\text {acc }}$ and $r_{\text {bur }}$ being the associated fractional amplitudes. The quantity $\Delta \varphi_{m}$ was measured earlier: $\Delta \varphi_{m} \lesssim 0.01$ cycles at the $99 \%$ confidence level. Using the values of $N_{\text {acc }}, N_{\text {bur }}, r_{\text {acc }}$ and $r_{\text {bur }}$ from Table 1 of W05, Equation (1) gives a $99 \%$ confidence upper limit on the bias introduced by any residual accretion pulsation of $10^{-3}$ cycles. This is sufficiently small that it does not affect our analysis. Similar conclusions can be reached for the overtone. Note that in computing these limits we assume that the accretion flow parameters $\left(N_{\text {acc }}, r_{\text {acc }}, \Delta \varphi_{m}\right)$ are unchanged during a burst. If accretion is inhibited during a burst, due to radiation pressure, the bias will be lower.

\section{DISCUSSION}

J1814 has unusual burst oscillation properties compared to other sources: they occur in hydrogenrich bursts, have negligible frequency and amplitude variation, and have a soft spectrum (S03, W05, Watts \& Strohmayer 2006). Our analysis has shown that the burst oscillations are also phase-locked to the accretion-powered pulsations (to within $3^{\circ}$ at $99 \%$ confidence) despite the substantial phase-wander exhibited by the latter over the course of the outburst.

In fact the burst oscillations are not only phase-locked but also coincident, having the same phase as the soft (2.5-5 keV), lagging, part of the accretion-powered pulsations (although they are also at the $2 \sigma$ level coincident with the entire 2.5-20 keV accretion-powered pulse). Detailed modeling of the accretion pulsations has yet to be done for J1814, but modeling for other AMXPs with similar pulse properties suggests that the soft pulsed component comes from a hot spot on the stellar surface, with the hard component originating in the accretion funnel (Poutanen \& Gierliński 2003; Gierliński \& Poutanen 2005; Falanga \& Titarchuk 2007).

We first consider what the exceptional degree of phaselocking implies about the cause of the variation in the TOA residuals. There are several parts of the system whose variation might affect both types of pulsation: surface rotation, the accretion funnel/disk, the magnetic field, and the fuel deposition footprint.

Case 1: Genuine changes in the spin rate of the stellar surface. Our result would be consistent with a model where all of the variation is due to spin changes, both sets of pulsations being locked to the surface. However this requires alternating spin-up and spin-down with $|\dot{\nu}| \sim 10^{-12} \mathrm{~Hz} / \mathrm{s}$. Even if the crust were decoupled from the fluid core, this is high compared to what is achievable from the expected accretion or gravitational wave torques (Andersson et al. 2005; Bildsten 1998a). Fitting a constant spin-down term $\dot{\nu} \approx 6 \times 10^{-14} \mathrm{~Hz} / \mathrm{s}$, as argued by $\mathrm{P} 07$, does improve the quality of the fits somewhat. However spin derivatives $|\dot{\nu}| \sim 10^{-12} \mathrm{~Hz} / \mathrm{s}$ would still be required on shorter timescales to explain the remaining excursions. Our results would also be consistent with precession, but modeling by Chung et al. (2008) suggests that precession is unlikely in this source.

Case 2: Changes in beaming/scattering by the accretion funnel or disk. The accretion shock in the funnel is thought to contribute to the pulsed emission of the accretion-powered pulsations, leaving a signature of hard emission in the spectrum. If the funnel were to have a similar effect on the much stronger burst emission it would have to do this without leaving any trace in the spectrum (Strohmaver et al. 2003; Krauss et al. 2005). This does not seem feasible. Our result also casts doubt on the accretion disk being the source of the soft lagging component of the accretion-powered pulsations (one of the possibilities considered by Falanga \& Titarchuk 2007), since it is hard to understand why the burst oscillations (a surface process) would track a component generated in the surrounding environs.

Case 3: Wander of the magnetic pole, or changes in field geometry. Motion of the magnetic pole would affect location of the accretion hot spot. If the magnetic field also determines the location of the nuclear burning hot spot through modulation of ignition or emission, this could also explain our result. However, the observed variability would require localized burial or amplification of the poloidal field component on timescales of order a day. The accretion rate in the peak of the outburst is at most a few percent of the Eddington rate (Galloway et al. 2004). Current modeling suggests that this is insufficient to cause burial of the polar field on the required timescales (Brown \& Bildsten 1998; Cumming et al. 2001; Pavne \& Melatos 2004). There is also no obvious mechanism for field amplification: material arriving via a funnel flow will have almost no angular momentum differential compared to the stellar surface. The heating associated with accretion could bring a buried field to the surface (Cumming et al. 2001), but as previously stated burial is unlikely at such low accretion rates.

Case 4: Wander of the fuel deposition point around the magnetic pole. Simulations of funneled accretion have shown that the fuel deposition point can exhibit phase excursions around a fixed magnetic pole as accretion rate fluctuates, particularly for small misalignment angles between the magnetic pole and the spin axis (Romanova et al. 2003, 2004; Lamb et al. 2008) $)^{2}$. Such a model might neatly account for the correlation between

2 The pulse profile modeling for this source which has been attempted has suggested large misalignment angles (Bhattacharvya et al. 2005; Leahy et al. 2008). If this were the case, the observed phase variability would require the fuel impact point to migrate back and forth by several $\mathrm{km}$ over the course of the outburst - an uncomfortably large amount. It seems more likely that some of the assumptions in these models, particularly the use of single temperature circular hotspots (known to be problematic, see Watts \& Strohmaver 2006), need to be revisited. 
residuals and flux without requiring any non-zero $\dot{\nu}$, since the stable position for the fuel impact point will vary in azimuth depending on accretion rate. The fact that the anti-correlation between flux and residuals is stronger when we set $\dot{\nu}=0$ supports this idea.

If the most plausible explanation for the variability in the two sets of pulsations is the last, what physical mechanisms might lead to phase-locking between the fuel impact point - which moves with accretion rate relative to field geometry on timescales of a few days - and the nuclear burning hot spots?

One possibility is some degree of magnetic confinement, leading to accumulation of fuel at the accretion hot spot. Material deposited near the polar cap will be prevented from spreading until the over-pressure is sufficient to distort the field lines (Brown \& Bildsten 1998), even if the impact point is not precisely on the polar cap. To ensure that the magnetic propeller effect does not disrupt accretion, the magnetic field of J1814 must be $\lesssim 10^{9}$ G (Psaltis \& Chakrabarty 1999; Rappaport et al. 2004). This is consistent with the upper limit on spin-down inferred from pulse timing (P07). At this upper limit helium could be confined until a column depth $\sim 10^{6} \mathrm{~g}$ $\mathrm{cm}^{-2}$, but this is still well before the material reaches the bursting layer at column depth $\sim 10^{8} \mathrm{~g} \mathrm{~cm}^{-2}$ (Bildsten 1998b). Hydrogen will spread even more easily. Based on these estimates we conclude that fuel confinement is not effective, although it has been advanced as a possible explanation for the short burst recurrence times (Galloway et al. 2004).

The other factor distinguishing the fuel impact point is its temperature, which is higher than the rest of the star. The magnitude of the temperature differential has not been determined observationally, but it could certainly affect burst emission. One possibility is that the higher temperatures modify the local composition (via steady burning between bursts, for example). The higher starting temperature and/or composition could in principle modify the flux from the burst once it starts; perhaps driving more energetic reactions or enhancing convection. Whether this effect would be large enough to explain the high fractional amplitudes is, however, not clear.

An alternative is the effect that a higher local temperature would have on ignition conditions. Previous studies that concluded that ignition would occur predominantly near the equator (Spitkovsky et al. 2002; Cooper \& Naravan 2007) did not consider the effect of non-uniformities in temperature. A small increase in temperature can have a large effect on the column depth required for ignition, with the hotter area requiring a lower column depth (Bildsten 1998b). Material at the fuel impact point could therefore reach ignition conditions well ahead of the rest of the star. In this scenario the burning front might stall before spreading across the whole star, depending on the rate of heat transfer across the burning front. This would result in a brightness asymmetry centered on the fuel impact point. Premature ignition at the fuel deposition point, followed by stalling, could explain several observational features: the rather small black body radius of the bursts (Galloway et al. 2006), the shorter than expected burst recurrence times (Galloway et al. 2004), and the burst shapes, which suggest off-equatorial ignition (Maurer \& Watts 2008). Note that off-equatorial ignition alone is not sufficient to explain the presence of an asymmetry in the burst tail: something else, such as stalling, is required.

Whatever the mechanism, it must fail once the accretion rate drops (or perhaps when the burst is more energetic), since the oscillations in the final burst are offset. The fuel impact footprint is by this time thought to be smaller, since the fractional amplitude of the accretionpowered pulsations is rising (W05). In addition the accreted fuel has more time to spread and equilibrate between bursts. Both factors will act to reduce the temperature differential between the fuel stream impact point and the rest of the star, which would make ignition at the fuel impact point less likely. The oscillation properties of this final burst (substantially lower fractional amplitude, frequency drift) are very different to the rest of the sample, and it is quite plausible that a different burst oscillation mechanism operates in this final burst.

We thank Mariano Mendez and the participants of the workshop "A Decade of Accreting Millisecond X-ray Pulsars" for the lively discussions that prompted and informed this work.

\section{REFERENCES}

Andersson N. et al., 2005, MNRAS, 361, 1153

Bhattacharyya S. et al., 2005, ApJ, 619, 483

Bildsten L., 1998, ApJ, 501, L89

Bildsten L., 1998, in The Many Faces of Neutron Stars, eds

Buccheri et al., Kluwer Academic Publishers, p.419

Brown E.F., Bildsten L., 1998, ApJ, 496, 915

Burderi L. et al., 2006, ApJ, 653, L133

Burderi L. et al., 2007, ApJ, 657, 961

Chakrabarty D. et al., 2003, Nature, 424, 42

Chung C. et al., MNRAS in press, eprint arXiv:0808.3820

Cooper R.L., Narayan R., 2007, ApJ, 657, L29

Cumming A. et al., 2001, ApJ, 557, 958

Falanga M., Titarchuk L., 2007, ApJ, 661, 1084

Galloway D.K. et al., 2002, ApJ, 576, L137

Galloway D.K. et al., 2004, BAAS, 36, 954

Galloway D.K. et al., ApJS in press, astro-ph/0608259

Gierliński M., Poutanen J., 2005, MNRAS, 359, 1261

Hartman J.M. et al., 2008, ApJ, 675, 1468

Jahoda K. et al., 2006, ApJSS, 163, 401

Krauss M.I. et al., 2005, ApJ, 627, 910

Lamb F.K. et al., eprint arXiv:0808.4159
Leahy D.A. et al., eprint arXiv:0806.0824

Markwardt C.B., Swank J.H., 2003, IAU Circ. 8144, 1

Markwardt C.B. et al., 2003, Atel 164.

Maurer I., Watts A.L., 2008, MNRAS, 383, 387

Muno M.P. et al., 2002, ApJ, 580, 1048

Payne D.J.B., Melatos A., 2004, MNRAS, 351, 569

Papitto A. et al., 2007, MNRAS, 375, 971

Papitto A. et al., 2008, MNRAS, 383, 411

Poutanen J., Gierliński M., 2003, MNRAS, 343, 1301

Psaltis D., Chakrabarty D., 1999, ApJ, 521, 332

Rappaport S.A. et al., 2004, ApJ, 606, 436

Riggio A. et al., 2008, ApJ, 678, 1273

Romanova M.M. et al., 2003, ApJ, 595, 1009

Romanova M.M. et al., 2004, ApJ, 610, 920

Rots A.H. et al., 2004, ApJ, 605, L129

Spitkovsky A. et al., 2002, ApJ, 566, 1018

Strohmayer T.E. et al., 2003, ApJ, 596, L67

Strohmayer T., Bildsten L., 2006, in Compact stellar X-ray

sources, eds Lewin W., van der Klis M., Cambridge

Astrophysics Series 39, Cambridge, UK, p.113 
Taylor J.H., 1993, in Pulsars as Physics Laboratories, ed. R.D.Blandford (New York: Oxford Univ. Press), 117

Watts A.L. et al., 2005, ApJ, 634, 547
Watts A.L., Strohmayer T.E., 2006, MNRAS, 373, 769 